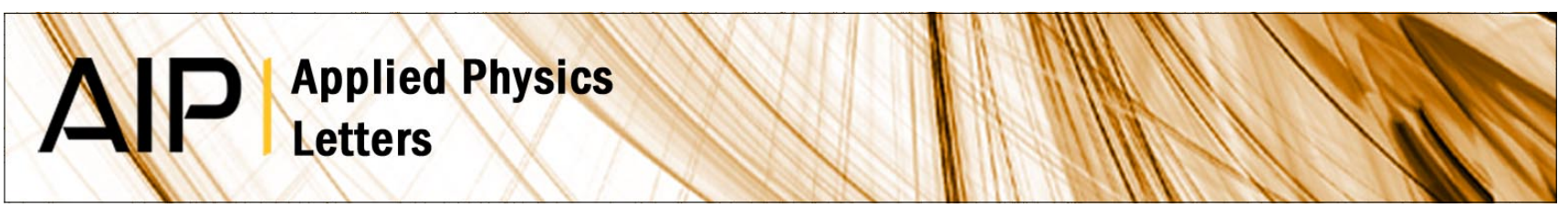

\title{
Enhanced spin signal in nonlocal devices based on a ferromagnetic CoFeAl alloy
}

G. Bridoux, M. V. Costache, J. Van de Vondel, I. Neumann, and S. O. Valenzuela

Citation: Appl. Phys. Lett. 99, 102107 (2011); doi: 10.1063/1.3635391

View online: http://dx.doi.org/10.1063/1.3635391

View Table of Contents: http://apl.aip.org/resource/1/APPLAB/v99/i10

Published by the American Institute of Physics.

\section{Related Articles}

Magnetic anisotropy and stacking faults in Co and Co84Pt16 epitaxially grown thin films J. Appl. Phys. 110, 093919 (2011)

Room temperature magnetoelectric memory cell using stress-mediated magnetoelastic switching in nanostructured multilayers

Appl. Phys. Lett. 99, 192507 (2011)

Composition dependence of magnetoresistance effect and its annealing endurance in tunnel junctions having $\mathrm{Mn}-\mathrm{Ga}$ electrode with high perpendicular magnetic anisotropy

Appl. Phys. Lett. 99, 192509 (2011)

Wide frequency range magnetoimpedance in tri-layered thin NiFe/Ag/NiFe films: Experiment and numerical calculation

J. Appl. Phys. 110, 093914 (2011)

Spin wave resonance detection using magnetic tunnel junction structure

Appl. Phys. Lett. 99, 192501 (2011)

\section{Additional information on Appl. Phys. Lett.}

Journal Homepage: http://apl.aip.org/

Journal Information: http://apl.aip.org/about/about_the_journal

Top downloads: http://apl.aip.org/features/most_downloaded

Information for Authors: http://apl.aip.org/authors

\section{ADVERTISEMENT}

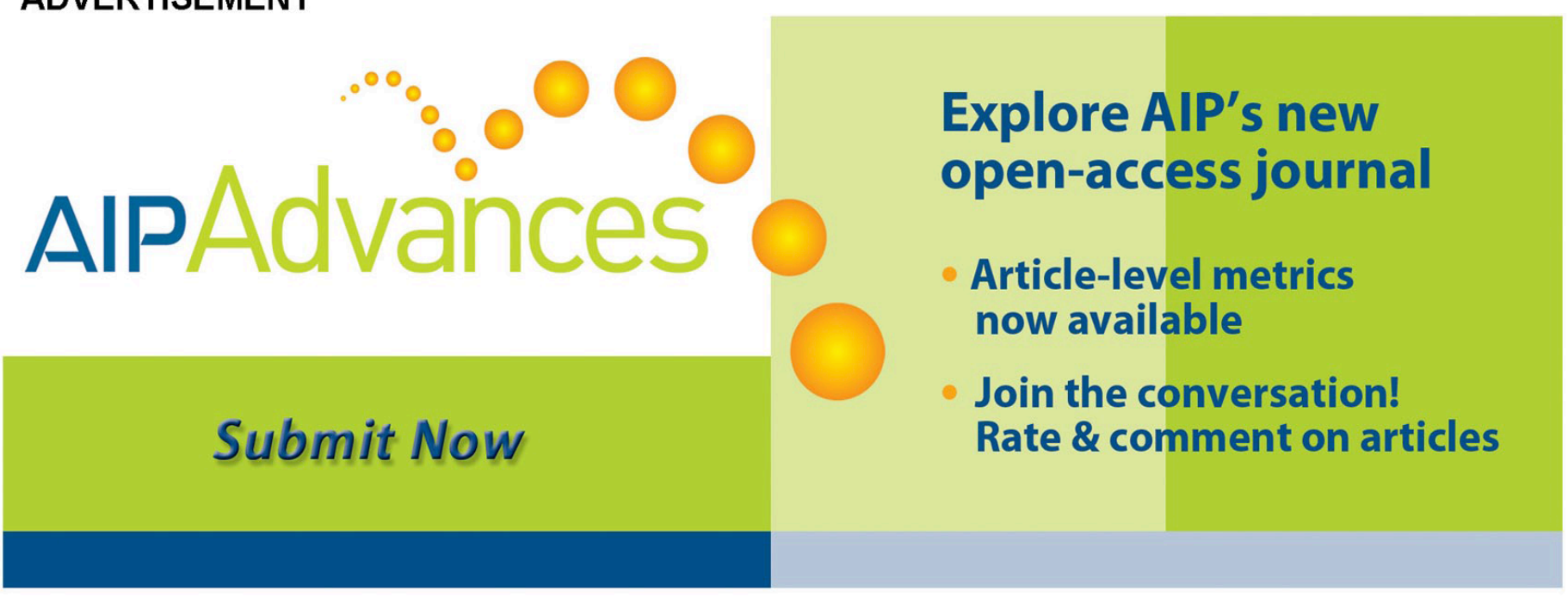




\title{
Enhanced spin signal in nonlocal devices based on a ferromagnetic CoFeAl alloy
}

\author{
G. Bridoux, ${ }^{1, a)}$ M. V. Costache,${ }^{1}$ J. Van de Vondel, ${ }^{1}$ I. Neumann,,${ }^{1}$ and S. O. Valenzuela ${ }^{1,2, b)}$ \\ ${ }^{1}$ Catalan Institute of Nanotechnology (ICN-CIN2), Bellaterra 08193, Spain \\ ${ }^{2}$ Institució Catalana de Recerca i Estudis Avançats (ICREA), Barcelona 08010, Spain and Universitat \\ Autonóma de Barcelona, Bellaterra 08193, Spain
}

(Received 26 April 2011; accepted 14 August 2011; published online 9 September 2011)

\begin{abstract}
We systematically study the nonlocal spin signal in lateral spin valves based on CoFeAl injectors and detectors and compare the results with identically fabricated devices based on CoFe. The devices are fabricated by electron beam evaporation at room temperature. We observe a $>10$-fold enhancement of the spin signal in the CoFeAl devices. We explain this increase as due to the formation of a highly spin-polarized $\mathrm{Co}_{2} \mathrm{Fe} \mathrm{Al}$ Heusler compound with large resistivity. These results suggest that Heusler compounds are promising candidates as spin polarized electrodes in lateral spin devices for future spintronic applications. (C) 2011 American Institute of Physics. [doi:10.1063/1.3635391]
\end{abstract}

The efficient injection, transport, and detection of spin polarized electrons are key elements in the development of spintronics, which is an emergent field aiming at enhancing electronics performance by taking advantage of the spin degrees of freedom. Nonlocal spin valves (NLSVs) (Refs. 1-4) comprising ferromagnetic (FM) and normal (N) electrodes provide a powerful tool to study spin transport and are increasingly used to gather information of the interface properties between FM and N, which determine the efficiency of spin injection and detection processes. ${ }^{4}$ NLSVs have also practical applications as part of spin torque ${ }^{5}$ and spin logic devices, ${ }^{6}$ if large spin accumulation is attained. In NLSVs, a pure spin current is generated by an electric current $I$ applied in the FM injector (Fig. 1(a)). The spin accumulation is then determined from a nonlocal voltage $V$ using a detector consisting of a second ferromagnet ${ }^{1,2,4}$ or a Hall cross. ${ }^{3}$ Once the spin transport properties of $\mathrm{N}$ are known, it is possible to directly study the interface effects. For example, these devices allow for spin-resolved tunneling spectroscopy measurements ${ }^{4,9}$ without the temperature and voltage bias constrains of classical methods, ${ }^{7}$ and have been recently used to demonstrate improved spin injection after the formation of a native oxide in $\mathrm{N},{ }^{10}$ or when inserting low-resistance $\mathrm{MgO}$ barriers, ${ }^{11}$ at the $\mathrm{FM} / \mathrm{N}$ interface.

Heusler alloys present a diverse range of magnetic properties, including ferromagnetic shape memory effects ${ }^{12}$ and half metallicity. ${ }^{13}$ The latter is supported by band structure calculations, which show that some Heusler compounds exhibit $100 \%$ spin polarization at the Fermi level. ${ }^{12,13}$ For this reason, giant magnetoresistance (GMR) and tunnel magnetoresistance devices have been implemented with them. ${ }^{14}$ However, annealing at temperatures higher than $300^{\circ} \mathrm{C}$, which is required to obtain an ordered crystalline Heusler phase, is incompatible with reliable device fabrication. Therefore, some studies focused on the properties of GMR devices based on $\mathrm{CoFe}$ compounds with added $\mathrm{Al}$, and annealing at lower temperatures. ${ }^{15}$ There, results show an

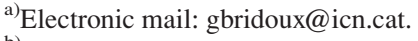

${ }^{\mathrm{b})}$ Electronic mail: SOV@icrea.cat.
}

increase in the overall GMR signal due to the addition of Al. Such an increase has been ascribed to an increase of the resistivity of the magnetic layers, ${ }^{15}$ while recent nuclear magnetic resonance studies also point to the formation of a highly spin-polarized $\mathrm{Co}_{2} \mathrm{FeAl}$ Heusler type structure on a local scale. ${ }^{16}$ Both properties are of importance to increase the signal in NLSVs.

In the present work, we systematically compare the spin signal of NLSVs based on CoFe with added Al with that of NLSVs based on CoFe, which is used as a reference material to independently study the spin properties of these compounds. We observe that the addition of $\mathrm{Al}$ results in a 10fold enhancement of the spin signal, making $\mathrm{CoFeAl}$ a very efficient spin injector/detector. Because of the simple room temperature deposition of $\mathrm{CoFeAl}$, this compound can become relevant for spintronics application in lateral devices. We ascribe our observations to the formation of a highly resistive, highly spin-polarized $\mathrm{CoFeAl}$ compound, which supports the hypothesis of the formation of a Heusler structure.

We fabricated a set of each $\mathrm{CoFeAl} / \mathrm{Al}$ and $\mathrm{CoFe} / \mathrm{Al}$ NLSVs with electron-beam lithography and shadow mask electron-beam evaporation. ${ }^{17,18}$ A scanning electron microscope (SEM) image of a typical device is shown in Fig. 1(a). In a first step (Fig. 1(b)), we evaporated the ferromagnetic electrodes from one side with a thickness of $15 \mathrm{~nm}$, and widths of $150 \mathrm{~nm}$ (FM1) and $110 \mathrm{~nm}$ (FM2). After this, without breaking the vacuum, we deposited an aluminium strip in two steps from two sides $(20 \mathrm{~nm}$ from each side, $40 \mathrm{~nm}$ total thickness, and $175 \mathrm{~nm}$ width) (Fig. 1(c)). For the CoFe NLSVs, we chose (Co:84, Fe:16 wt. \%) because it is widely recognized as having one of the highest spin polarizations among the $3 d$ ferromagnet alloys ${ }^{19}$ while for the CoFeAl ones, we used (Co:36, Fe:45, Al:19 wt. \%) as determined by energy-dispersive x-ray spectroscopy (EDX). The chosen composition of $\mathrm{CoFeAl}$ is near the optimum value obtained from GMR measurements. ${ }^{15,16}$ All of the devices in the present study have the same thicknesses and widths for FM1, FM2, and Al, whereas the center to center distance $d$ between the FMs was varied from $400 \mathrm{~nm}$ to $850 \mathrm{~nm}$. The 
(a)
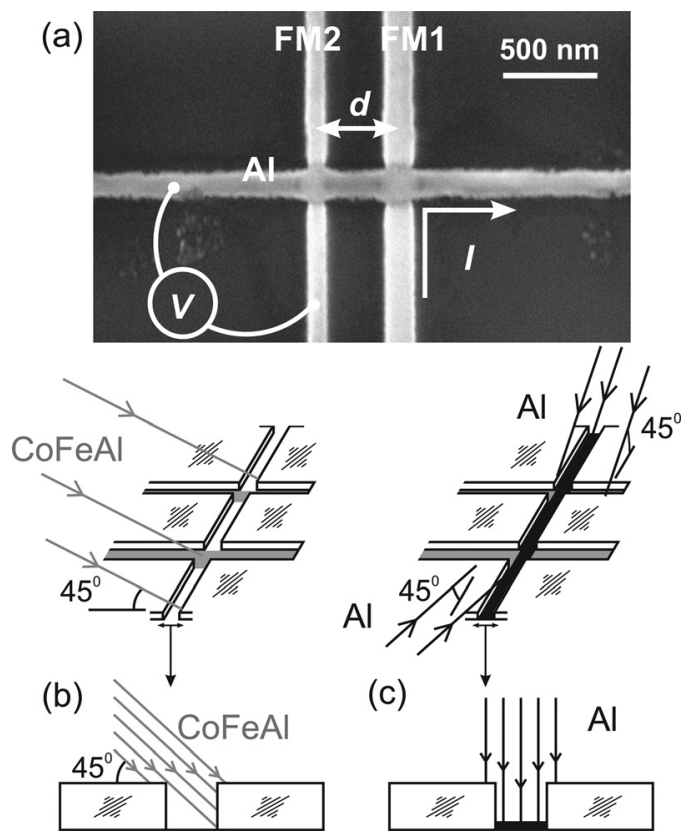

FIG. 1. (a) SEM image of a typical NLSV and measurement setup. The magnetic field, $\mu_{0} H$, is applied parallel to the FMs axis. (b) Sketch of the FM electrodes evaporation and (c) the subsequent aluminium strip evaporation. The dashed regions represent the electron-beam resist.

transport measurements were done using a standard lock-in technique, injecting a current $I=50 \mu \mathrm{A}$, and measuring a voltage $V$ at the detector (Fig. 1(a)).

Figure 2 shows typical measurements of the nonlocal spin transresistance, $V / I$, as a function of the magnetic field, $\mu_{0} H$, for geometrically identical $\mathrm{CoFe}$ (red triangles) and $\mathrm{CoFeAl}$ (blue circles) NLSVs at $T=77 \mathrm{~K}$ and with $d=400 \mathrm{~nm}$. The magnetic field is applied along the ferromagnetic electrodes. When it is swept from negative to positive, a change in the sign of the detector signal is observed when the magnetization of one of the electrodes reverses, and the device magnetization configuration switches from parallel to antiparallel or vice versa. Remarkably, we observe that the change in $V /$ $I, \delta R$, is larger by more than one order of magnitude for the CoFeAl-based device, for which $\delta R \sim 12 \mathrm{~m} \Omega$, when compared to the value for the CoFe-based one, where $\delta R \sim 0.78 \mathrm{~m} \Omega$

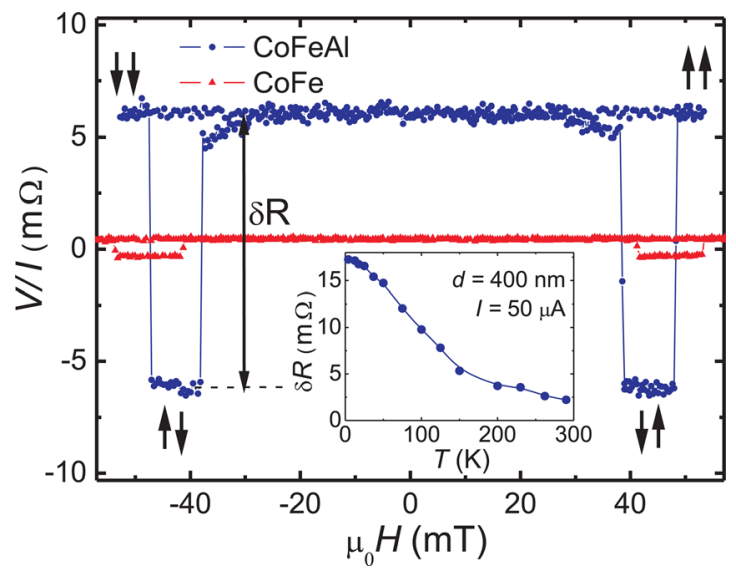

FIG. 2. (Color online) Typical nonlocal spin valve signal, $V / I$, as a function of $\mu_{0} H$, for NLSVs based on $\mathrm{CoFeAl}$ (blue circles) and $\mathrm{CoFe}$ (red triangles) at $T=77 \mathrm{~K}(I=50 \mu \mathrm{A}$ and $d=400 \mathrm{~nm})$. Inset: temperature dependence of the nonlocal spin valve transresistance change $\delta R$ for a CoFeAl based NLSV $(I=50 \mu \mathrm{A}$ and $d=400 \mathrm{~nm})$. (the inset of Fig. 2 shows the temperature dependence of $\delta R$, which presents an overall decrease similar to that observed in Ag devices ${ }^{10}$ ).

In order to determine the origin of the signal enhancement when using $\mathrm{CoFeAl}$, we have compared $\delta R$ as a function of $d$ for the CoFeAl and CoFe set of devices (Fig. 3) and modeled the observed values using a 1D spin-dependent diffusion equation $^{20}$ solved for the NLSV geometry ${ }^{21}$ for transparent contacts

$$
\delta R=\frac{2 P_{F M}^{2} R_{A l}}{\left(2+\frac{R_{A l}}{R_{F M}}\right)^{2} e^{d / \lambda_{A l}}-\left(\frac{R_{A l}}{R_{F M}}\right)^{2} e^{-d / \lambda_{A l}}},
$$

where $P_{F M}$ is the spin polarization of the FM electrodes (CoFe or $\mathrm{CoFeAl}$ ). $R_{A l}$ and $R_{F M}$ are the so-called spin resistances of the $\mathrm{Al}$ and $\mathrm{FM}(\mathrm{CoFe}$ or $\mathrm{CoFeAl})$ thin films, respectively, which are defined as $R_{A l}=2 \rho_{A l} \lambda_{A l} / S_{A l}$ and $R_{F M}=2 \rho_{F M} \lambda_{F M} / S_{F M}\left(1-P_{F M}^{2}\right)$ with $\lambda_{A l, F M}, \rho_{A l, F M}$, and $S_{A l, F M}$ being the spin-diffusion lengths, the resistivities, and the effective cross-sectional areas of the Al strip and the FM electrodes, respectively. The assumption of transparent contacts, which is a very reasonable given the fact that we fabricate the full device structure without breaking vacuum, was verified by means of four probe resistance measurements.

As expected from Eq. (1), $\delta R$ decreases nearly exponentially as a function of $d$ with the same characteristic length $\lambda_{A l}$ for the two sets of samples, which is evident in the semilogarithmic plot of Fig. 3. This proves that the signal enhancement observed in the CoFeAl devices is directly related to the ferromagnetic material and not to the spin diffusion properties of $\mathrm{Al}$, thus a reliable comparison with the spin injection properties of CoFe can be done. By fitting the data to Eq. (1), we obtain $P_{F M}$. For this purpose, we use the resistivities $\rho_{A l}=8 \times 10^{-8} \Omega \mathrm{m}, \rho_{\text {CoFeAl }}=4.2 \times 10^{-6} \Omega \mathrm{m}$, and $\rho_{\mathrm{CoFe}}=2.3 \times 10^{-7} \Omega \mathrm{m}$, as measured with standard four probe techniques, and the spin-diffusion lengths $\lambda_{\text {CoFeAl }}=(3.0 \pm 0.5) \mathrm{nm}^{15}$ and $\lambda_{\text {CoFe }}=(11.0 \pm 0.1) \mathrm{nm}^{22}$ Note that the spin diffusion length of our CoFeAl films is likely overestimated and is probably smaller than $2 \mathrm{~nm}$ because the resistivity of our films is more than 3 times larger than that of the films in Ref. 15.

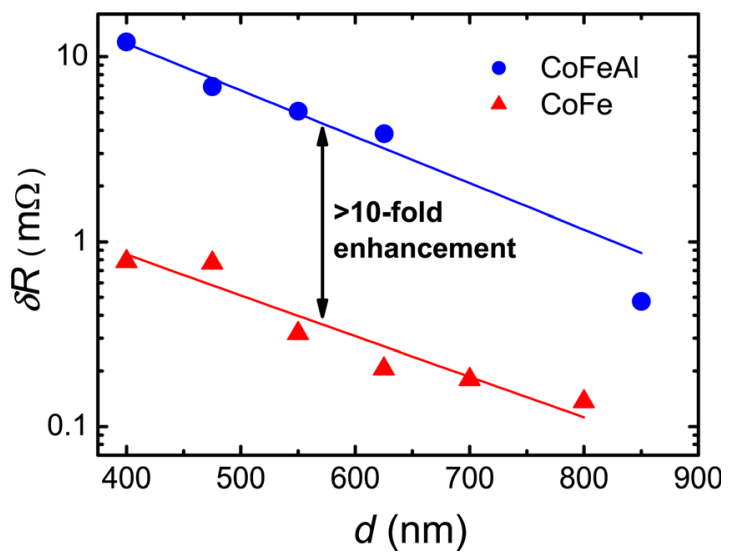

FIG. 3. (Color online) Nonlocal spin valve resistance, $\delta R$, as a function of the distance $d$ between the FMs at $T=77 \mathrm{~K}(I=50 \mu \mathrm{A})$. The blue circles correspond to the CoFeAl based NLSVs and the red triangles to the CoFe based ones. The lines are the corresponding fittings to Eq. (1). 
TABLE I. Spin injection/detection efficiency $\gamma$ in metallic NLSVs.

\begin{tabular}{lccc}
\hline \hline Materials FM/N & $T[\mathrm{~K}]$ & $\gamma\left[\left(\mathrm{m} \Omega \mu \mathrm{m}^{2}\right)^{2}\right]$ & Reference \\
\hline $\mathrm{CoFeAl} / \mathrm{Al}$ & 77 & $400 \pm 100$ & This work \\
$\mathrm{CoFe} / \mathrm{Al}$ & 77 & $9 \pm 2$ & This work \\
$\mathrm{NiFe} / \mathrm{Ag}$ & 77 & $6 \pm 1$ & 26 \\
$\mathrm{NiFe} / \mathrm{Ag}$ & 79 & $4.0 \pm 0.3$ & 24 \\
$\mathrm{NiFe} / \mathrm{Ag}$ & 77 & $1.34 \pm 0.02$ & 25 \\
$\mathrm{NiFe} / \mathrm{Au}$ & 10 & $0.85 \pm 0.02$ & 23 \\
$\mathrm{NiFe} / \mathrm{Cu}$ & 4.2 & $0.6 \pm 0.1$ & 27 \\
$\mathrm{Co} / \mathrm{Cu}$ & 10 & $0.46 \pm 0.05$ & 23 \\
$\mathrm{NiFe} / \mathrm{Cu}$ & 4.2 & $0.04 \pm 0.01$ & 2 \\
\hline \hline
\end{tabular}

The large difference between $\rho_{\mathrm{CoF} \text { Al }}$ and $\rho_{\mathrm{CoFe}}$ highly contributes to the improved spin injection properties of $\mathrm{CoFeAl}$. However, the fittings to Eq. (1) also point to an enhanced polarization with $P_{C o F e}=0.45 \pm 0.05$ and $P_{C o F e A l}=0.55 \pm 0.07$ for $\lambda_{\mathrm{CoF} e A l}=3 \mathrm{~nm}$. Given that we cannot measure $P_{\text {CoFeAl }}$ and $\lambda_{\mathrm{CoFeAl}}$ independently with our experiments, further studies are required to probe this tendency, in particular because there exists a small overlap between the two polarizations due to their uncertainties. However, considering $\lambda_{\mathrm{CoFeAl}} \approx 2 \mathrm{~nm}$ results in $P_{\text {CoFeAl }}=0.63 \pm 0.07$ making the difference significative.

The enhancement of the spin signal in the CoFeAl devices would thus be due to the combined effect of a resistivity and spin polarization increase, which supports the hypothesis of the local formation of a highly spin-polarized Heusler structure in this material. ${ }^{16} P_{\mathrm{CoFe}}$ is in agreement with previously reported values for $3 d$ ferromagnetic alloys in ohmic NLSVs and is close to the spin polarization reported for $\mathrm{CoFe}$ in $\mathrm{CoFe} / \mathrm{Al}_{2} \mathrm{O}_{3} /$ Al tunnel junctions, ${ }^{19}$ validating our analysis to extract $P_{F M}$.

Lastly, a comparison between the achieved spin injection/ detection efficiency in our ohmic NLSVs with other values in the literature ${ }^{2,23-27}$ can be done by defining the parameter $\gamma=\left(P_{F M} R_{F M} S_{F M}\right)^{2}$, which is a dominant contribution in Eq. (1) and is independent of geometrical factors and the normal metal properties. The spin injection/detection efficiency is directly proportional to $\gamma$ when $R_{N} \gg R_{F M}$, a scenario commonly found in NLSVs. This parameter is thus an important figure of merit for technological application of NLSVs. The results are summarized in Table I and show that the CoFeAl electrodes bring about a significant spin signal improvement, usually in the range of one to two orders of magnitude.

In conclusion, we have implemented $\mathrm{CoFeAl}$ electrodes in NLSVs. A comparative study with CoFe NLSVs shows a $>10$-fold enhancement of the spin signal in the CoFeAl spin valves that results from a larger resistivity and, very likely, a larger spin polarization in $\mathrm{CoFeAl}$ than in $\mathrm{CoFe}$. The high spin polarization suggests the formation of a highly spinpolarized Heusler compound on a local scale and underscores the importance of these compounds for future spintronics devices, where efficient spin injection using ohmic contacts is desirable to reduce dissipation. In addition, high-resistivity Heusler compounds can prove useful for spin injection into low conductance materials, such as graphene about the Dirac point, where the conductivity mismatch seriously degrades the spin injection efficiency.

We thank B. Ballesteros for the EDX measurements. We acknowledge the financial support from the Spanish Ministerio de Ciencia e Innovación, MICINN (MAT201018065, FIS2009-06671-E, and GICSERV program “Access to ICTS integrated nano- and microelectronics cleanroom"). J.V.d.V. acknowledges the support from FWO-VL.

${ }^{1}$ M. Johnson and R. H. Silsbee, Phys. Rev. Lett. 55, 1790 (1985); Phys. Rev. B 35, 4959 (1987); M. Johnson and R. H. Silsbee, ibid. 37, 5236 (1988).

${ }^{2}$ F. J. Jedema, A. T. Filip, and B. J. van Wees, Nature (London) 410, 345 (2001).

${ }^{3}$ S. O. Valenzuela and M. Tinkham, Nature (London) 442, 176 (2006).

${ }^{4}$ S. O. Valenzuela, Int. J. Mod. Phys. B 23, 2413 (2009).

${ }^{5}$ T. Yang, T. Kimura, and Y. Otani, Nat. Phys. 9, 851 (2008).

${ }^{6}$ H. Dery, P. Dalal, Ł. Cywiński, and L. J. Sham, Nature 447, 573 (2007).

${ }^{7}$ R. Meservey and P. M. Tedrow, Phys. Rep. 238, 173 (1994).

${ }^{8}$ F. J. Jedema, H. B. Heersche, A. T. Filip, J. J. A. Baselmans, and B. J. van Wees, Nature (London) 416, 713 (2002).

${ }^{9}$ S. O. Valenzuela, D. J. Monsma, C. M. Marcus, V. Narayanamurti, and M. Tinkham, Phys. Rev. Lett. 96, 196601 (2005).

${ }^{10}$ G. Mihajlovic, D. K. Schreiber, Y. Liu, J. E. Pearson, S. D. Bader, A. K. Petford-Long, and A. Hoffmann, Appl. Phys. Lett. 97, 112502 (2010).

${ }^{11}$ Y. Fukuma, L. Wang, H. Idzuchi, and Y. Otani, Appl. Phys. Lett. 97, 012507 (2010).

${ }^{12}$ B. L. Ahuja, B. K. Sharma, S. Mathur, N. L. Heda, M. Itou, A. Andrejczuk, Y. Sakurai, A. Chakrabarti, S. Banik, A. M. Awasthi, and S. R. Barman, Phys. Rev. B 75, 134403 (2007).

${ }^{13}$ R. A. de Groot, F. M. Mueller, P. G. van Engen, and K. H. J. Buschow, Phys. Rev. Lett. 50, 2024 (1983).

${ }^{14}$ S. Okamura, A. Miyazaki, S. Sugimoto, N. Tezuka, and K. Inomata, Appl. Phys. Lett. 86, 232503 (2005); W. Wang, H. Sukegawa, R. Shan, S. Mitani, and K. Inomata, Appl. Phys. Lett. 95, 182502 (2009), in references therein.

${ }^{15}$ S. Maat, M. J. Carey, and J. R. Childress, J. Appl. Phys. 101, 093905 (2007).

${ }^{16}$ S. Wurmehl, P. J. Jacobs, J. T. Kohlhepp, H. J. M. Swagten, B. Koopmans, S. Maat, M. J. Carey, and J. R. Childress, Appl. Phys. Lett. 98, 012506 (2011).

${ }^{17}$ S. O. Valenzuela and M. Tinkham, J. Appl. Phys. 101, 09B103 (2007).

${ }^{18}$ M. V. Costache and S. O. Valenzuela, Science 330, 1645 (2010).

${ }^{19}$ D. J. Monsma and S. S. P. Parkin, Appl. Phys. Lett. 77, 720 (2000).

${ }^{20}$ T. Valet and A. Fert, Phys. Rev. B 48, 7099 (1993).

${ }^{21}$ S. Takahashi and S. Maekawa, Phys. Rev. B 67, 052409 (2003).

${ }^{22}$ C. Ahn, K.-H. Shin, R. Loloee, J. Bass, and W. P. Pratt, Jr., J. Appl. Phys. 108, 023908 (2010).

${ }^{23}$ Y. Ji, A. Hoffmann, J. S. Jiang, and S. D. Bader, Appl. Phys. Lett. 85, 6218 (2004); Y. Ji, A. Hoffmann, J. E. Pearson, and S. D. Bader, ibid. 88, 052509 (2006).

${ }^{24}$ R. Godfrey and M. Johnson, Phys. Rev. Lett. 96, 136601 (2006).

${ }^{25}$ T. Kimura and Y. Otani, Phys. Rev. Lett. 99, 196604 (2007).

${ }^{26}$ G. Mihajlovic, J. E. Pearson, S. D. Bader, and A. Hoffmann, Phys. Rev. Lett. 104, 237202 (2010).

${ }^{27}$ M. Erekhinsky, A. Sharoni, F. Casanova, and I. K. Schuller, Appl. Phys. Lett. 96, 022513 (2010). 